\title{
Bone Segmentation and Fracture Detection in Ultrasound Using 3D Local Phase Features
}

\author{
Ilker Hacihaliloglu ${ }^{1}$, Rafeef Abugharbieh ${ }^{1}$, Antony Hodgson ${ }^{2}$, and Robert Rohling ${ }^{1,2}$ \\ ${ }^{1}$ Department of Electrical and Computer Engineering \\ ${ }^{2}$ Department of Mechanical Engineering, University of British Columbia, \\ Vancouver, BC, Canada \\ ilkerh@ece.ubc.ca, rafeef@ece.ubc.ca, ahodgson@mech.ubc.ca, \\ rohling@ece.ubc.ca
}

\begin{abstract}
D ultrasound (US) is increasingly considered as a viable alternative imaging modality in computer-assisted orthopaedic surgery (CAOS) applications. Automatic bone segmentation from US images, however, remains a challenge due to speckle noise and various other artifacts inherent to US. In this paper, we present intensity invariant three dimensional (3D) local image phase features, obtained using 3D Log-Gabor filter banks, for extracting ridge-like features similar to those that occur at soft tissue/bone interfaces. Our contributions include the novel extension of 2D phase symmetry features to 3D and their use in automatic extraction of bone surfaces and fractured fragments in 3D US. We validate our technique using phantom, in vitro, and in vivo experiments. Qualitative and quantitative results demonstrate remarkably clear segmentations results of bone surfaces with a localization accuracy of better than $0.62 \mathrm{~mm}$ and mean errors in estimating fracture displacements below $0.65 \mathrm{~mm}$, which will likely be of strong clinical utility.
\end{abstract}

Keywords: 3D ultrasound, local phase features, 3D Log-Gabor filters, 3D phase symmetry, bone segmentation.

\section{Introduction}

The two most common imaging modalities used in orthopaedic surgery are fluoroscopy (projection x-rays) and computed tomography (CT). Although both methods provide clear images of bone structures, CT normally cannot be performed intraoperatively and fluoroscopy typically produces two dimensional images only, which makes it difficult for surgeons to assess the 3D shape and position of bones and bone fragments (e.g. during reduction procedures). This is particularly important in complex fracture cases involving bones such as the distal radius, responsible for about one sixth of all fractures seen in emergency departments in the United States [1], and the pelvis [2]. Furthermore, both CT and fluoroscopy use ionizing radiation, which raises important safety concerns for both patients and surgeons.

Ultrasound (US) imaging is non-ionizing, fast, portable, inexpensive and capable of real time imaging, but, unfortunately, US images typically contain significant 
speckle and other artifacts which complicate image interpretation and automatic processing [3]. If anatomical structures of interest could be visualized and localized with sufficient accuracy and clarity, 3D US may in fact become a strong practical alternative imaging modality for selected applications in orthopaedic surgery, particularly for computer-assisted applications where the image can be processed to provide quantitative information on the location of bone structures.

Manual identification of bone surfaces in 2D US for orthopedic surgery application was reported in [4]. Manual processing, however, is time consuming and operator dependent, and thus limits clinical practicality. While some studies have shown some promise in automatically identifying the bone surface based on intensity and gradient information (or a combination of both) these techniques were limited to 2D US, and remained sensitive to typical image variability and choice of processing parameters [5]. Daanen et al [6] proposed a method where prior knowledge of bone appearance was incorporated. However, fractured bone surfaces do not have a continuous smooth surface and prior knowledge of fragment shape is mostly unavailable. Other approaches combined intensity and gradient-based techniques with multimodal registration of US to preoperative CT [7]. However, preoperative CT requires additional time and expense and is not always considered necessary for diagnosis or treatment, so it is only available in selected cases.

Local phase based features have been used in US image analysis, e.g. for localizing endocardial border points in echocardiography [8]. In [9], Hacihaliloglu et al proposed using phase features extracted automatically from 2D slices to identify bone boundaries in US data. However, 2D methods do not take advantage of correlations between adjacent images (i.e., along the axis perpendicular to the scan plane direction) and are therefore subject to spatial compounding errors as well as errors due to beam thickness effects. In this paper, we extend local phase based processing to 3D US volumes using 3D Log-Gabor filters. Specifically, we construct a 3D local phase symmetry measure which produces strong responses at bone surfaces and suppresses responses elsewhere. We quantitatively investigate the accuracy of our technique in localizing bone surfaces and assess the technique's ability to resolve displaced bone fragments. The current study is therefore the first to show that bone surfaces and fractures can be accurately localized using local phase features computed directly from 3D ultrasound image volumes.

\section{Methods}

In US images, bone surfaces typically appear blurry with non-uniform intensity and substantial shadowing beneath the surface. The thickness of the response at the leading edge ranges from 2-4 mm [3] for a typical transducer. In [3], it was shown that the actual bone surface lies between the highest gradient and the highest intensity points of this thick response. We propose that it would therefore be more appropriate to use a ridge detector, rather than an edge detector, to identify the bone surface location as the latter would produce responses on both sides of the band at the bone surface. 


\subsection{D Local Phase Symmetry Feature}

The purpose of ridge detection is to capture the major axis of symmetry of a feature at some specified spatial scale. Signals that have even symmetry about the origin will have real (and even) Fourier transforms, while signals that have odd symmetry will have imaginary (and odd) Fourier transforms. Signals that are neither perfectly odd nor perfectly even will have complex Fourier transforms (i.e. have both real and imaginary parts) where the resultant phase values reflects their degree of symmetry. Local phase information of a 1D signal can be obtained by convolving the signal with a pair of band-pass quadrature filters (an odd filter and an even filter). Using two filters in quadrature enables the calculation of signal amplitude and phase at a particular scale (spatial frequency) at a given spatial location. A good choice of quadrature filters is the Log-Gabor filter which can be constructed with arbitrary bandwidth. In order to obtain simultaneous localization of spatial and frequency information, analysis of the signal must be done over a narrow range (scale) of frequencies at different locations in the signal. This can be achieved by constructing a filter bank using a set of quadrature filters created from rescalings of the Log-Gabor filter. Each scaling is designed to pick out particular frequencies of the signal being analyzed. In [10] Kovesi investigated symmetry information by looking at the points where the response of the even filter dominates the response of the odd filter taking the difference of their absolute values. In this paper we extend this analysis to 3D using 3D LogGabor filters.

The transfer function $(G)$ of a 3D Log-Gabor filter in the frequency domain (1) is constructed as the product of two components: a one dimensional Log Gabor function that controls the frequencies to which the filter responds and a rotational symmetric angular Gaussian function that controls the orientation selectivity of the filter [11].

$$
G(\omega, \phi, \theta)=\exp \left(\frac{\left(\log \left(\omega / \omega_{0}\right)\right)^{2}}{2\left(\log \left(\kappa / \omega_{0}\right)\right)}\right) \times \exp \left(-\frac{\alpha\left(\phi_{i}, \theta_{i}\right)^{2}}{2 \sigma_{\alpha}^{2}}\right)
$$

Here $\kappa$ determines the bandwidth of the filter in the radial direction and $\omega_{0}$ is the filter's center spatial frequency. To achieve constant shape ratio filters, which are filters that are geometric scalings of a reference filter, the term $\kappa / \omega_{0}$ must be kept constant. The angle between the direction of the filter, which is determined by the azimuth $(\phi)$ and elevation $(\theta)$ angles, and the position vector of a given point $f$ in the frequency domain is given by $\alpha\left(\phi_{i}, \theta_{i}\right)=\operatorname{arcos}\left(\mathrm{f} . v_{\mathrm{i}} /\|f\|\right) . v_{\mathrm{i}}=\left(\cos \phi_{\mathrm{i}} \cos \theta_{\mathrm{i}}, \cos \phi_{\mathrm{i}} \sin \theta_{\mathrm{i}}, \sin \phi_{\mathrm{i}}\right)$ is a unit vector in the filter's direction. Here $\sigma_{\alpha}$ defines the extent of spreading in the angular direction. To get higher orientation selectivity, the angular function must become narrower.

The scaling of the radial Log Gabor function is achieved by using different wavelengths which are based on multiples of a minimum wavelength, $\lambda_{\min }$, which is a userdefinable parameter. The relationship between the filter scale $m$, and the filter center frequency $\omega_{0}$ is defined as $\omega_{0}=2 / \lambda_{\min } \times(\delta)^{\mathrm{m}-1}$ where $\delta=3$ is a scaling factor defined for computing the center frequencies of successive filters. After investigating convolution results of various 1D scanline profiles of a distal radius, scanned in vivo, with a pair of quadrature filters at different scales, selecting a single scale $(m=1)$ with a large wavelength $\left(\lambda_{\min }=25\right)$ gave well localized bone surface phase features. A value of $\kappa / \omega_{0}=0.25$ provided good surface localization in the presence of speckle. For the 
angular component, we found empirically after some experimentation with models of the human distal radius and pelvis that it was possible to get good orientation resolution while containing an adequate range of frequencies by selecting $\sigma_{\alpha}=14.3^{\circ}$. The filter bank used in this work uses 15 different $(\alpha)$ 3D filter orientations. Our local phase analysis of a 3D image volume $V(x, y, z)$ proceeds by convolving the image with the 3D Log Gabor filters. Let $M_{r m}^{e}\left(\omega, \phi_{r}, \theta_{r}\right)=\operatorname{real}\left(G\left(\omega, \phi_{r}, \theta_{r}\right)\right)$ and $M_{r m}^{o}\left(\omega, \phi_{r}, \theta_{r}\right)=$ $\operatorname{imag}\left(G\left(\omega, \phi_{r}, \theta_{r}\right)\right)$ denote the real (even) and imaginary (odd) parts, respectively, at a scale $m$ and orientation $r$, and let $\hat{H}(u, v t)$ be the Fourier transform of $V(x, y, z)$. We can think of the responses of each quadrature pair of filters as forming a response vector $\left[e_{r m}(x, y, z), o_{r m}(x, y, z)\right]=\left[\boldsymbol{F}^{-1}\left(\hat{H}(u, v, t) \mathbf{M}_{r m}^{e}\left(\omega, \phi_{r}, \theta_{r}\right)\right), \boldsymbol{F}^{-1}\left(\hat{H}(u, v, t) M_{r m}^{o}\left(\omega, \phi_{r}, \theta_{r}\right)\right)\right]$. Here $\boldsymbol{F}^{-1}$ denotes the inverse Fourier transform operation.

Extending Kovesi's work where 2D phase symmetry was defined as in [10], we construct a 3D phase symmetry measure, for different scales $(m)$ and orientations $(r)$ :

$$
3 D P S(x, y, z)=\frac{\left.\sum_{r} \sum_{m} \mid\left[\left|e_{r m}(x, y, z)\right|-\left|o_{r m}(x, y, z)\right|\right]-T_{r}\right\rfloor}{\sum_{r} \sum_{m} \sqrt{e_{r m}^{2}(x, y, z)-o_{r m}^{2}(x, y, z)}+\varepsilon}
$$

Here $\lfloor\mathrm{A}\rfloor=\max (\mathrm{A}, 0), \varepsilon$ is a small number included to avoid division by zero, and $T$ is a noise threshold calculated as a specified number $(k)$ of standard deviations $(\sigma)$ above the mean $(\mu)$ of the local energy distribution. Based on this, $T$ is defined as: $T=\mu+$ $k \times \sigma$ and the distribution is expected to be Rayleigh [12]. The response of the smallest scale filter is used for the calculation of $\mu$ and $\sigma$ since it has the largest bandwidth and will give the strongest noise response. For different US transducers and scales, $k$ can be tuned to provide a balance between the detected bone surface and speckle scale. The noise threshold parameter $k$ was set to 5 . Throughout our experiments the selected filter parameters were not changed.

\subsection{Experimental Setup}

We designed two different experiments to quantitatively evaluate the performance of the proposed 3D local phase-based bone segmentation method. The first experiment aimed at assessing the localization accuracy of our bone surface detection technique and the second at assessing the accuracy of measuring relative displacements between bone fragments, as this is a clinically relevant task for which we would like to use 3D US imaging. Acquisition was performed on a GE Voluson 730 Expert ultrasound machine (GE Healthcare, Waukesha, WI) with a 3D RSP5-12 probe. This is a mechanized probe where a linear array transducer is swept through an arc range of $20^{\circ}$. The reconstructed US volumes were $199 \times 119 \times 50$ voxels (lateral $\times$ axial $\times$ elevational) with an isotropic voxel size of $0.19 \mathrm{~mm}$. Our algorithm was implemented in MATLAB and run on an Intel Pentium 4 PC (3.64 GHz, 2GB of RAM). A human left radius Sawbone (Sawbones Inc., Vashon, WA), an in vitro pig leg and in vivo scans of a human distal radius were used in the validation experiments. In addition to our two quantitative studies, we also present qualitative results for an in vivo scan of a human distal radius and for an ex vivo scan of a porcine tibia and fibula. 
Experiment 1 Surface Localization: In order to quantify our bone localization accuracy, a stylus with a spherical tip was placed at a variety of locations on the surface of a bone or bone model. Two situations were assessed: (A) a plastic bone model (Sawbone) in a water tank, and (B) an ex vivo porcine specimen.

(A) Sawbone Specimen: Scans of the Sawbone model were performed inside a water tank with the long axis of the bone aligned with the axis of the linear array of the mechanized transducer. This alignment produced the clearest depiction of the bone surface. Images of the Sawbone were obtained at varying depths (shallow, middle, deep) by changing the probe position inside the water tank. Different depths produce different US resolutions in the elevation and lateral directions of the linear array. Realignment of the Sawbone was performed at each depth to ensure the bone was aligned with the probe's central slice. To test the accuracy of surface localization at different beam orientations relative to the bone surface, as might occur in clinical use, we used two different orientations for the phantom - horizontal and inclined at $20^{\circ}$. At each probe position, the US volume acquisition was repeated with a stylus tipped with a small spherical bead $(3.0 \mathrm{~mm}$ in diameter) placed on the bone to provide the 'gold standard' for the true bone location. The bead was placed and scanned at 30 different locations along the bone surface for each of the 3 depth settings. To ensure that the bead was centered in the elevation direction, the position of the stylus was adjusted until the clearest surface reflection was obtained. The location of the dot-like bright intensity response which is the top of the bead tip, $f$, and the location of the intensity response of the bone surface obtained from the phase algorithm, $b$, were then extracted (Fig. 1a-c). These two measurements, $b$ and $f$, were obtained in a highly automated and repeatable manner using a subpixel edge detection algorithm. To ensure that the images with and without the bead in place were otherwise identical, the Sawbone and probe were both fixed for both image acquisitions. The bone surface localization error was therefore defined as: error $=D(b f)$, where $D$ is the bead diameter and $b$ and $f$ are expressed in $\mathrm{mm}$. When the US beam was not perpendicular to the bead on the bone surface, the position of the underlying bone surface was compensated for using the geometry calculations shown in Figure 1c. Since mechanized 3D ultrasound probes use a set of 2D images to reconstruct a volume, the effect of the finite beam thickness is incorporated into the volume data via the reconstruction process. Compared to a single 2D image, volumetric data provides information about the bone response away from a single plane, so the analysis implicitly includes beam thickness effects.

(B) Ex Vivo Porcine Specimen: In order to investigate the effect of a soft tissue interface on our localization accuracy, we conducted an ex vivo experiment on a porcine tibia and fibula. First, the soft tissue was removed of the bone and the same spherical bead-tipped stylus described above was placed against the bone. The removed soft tissue was then overlaid, leaving the bead underneath the tissue and touching the bone. Again, a set of 3D scans were obtained with the bead positioned at 30 different locations along the bone surface. The error calculation proceeded in the same manner described in part (A) above.

Experiment 2 Fracture Misalignment Detection: Identification of fractures and proper assessment of fracture reduction is of special importance in orthopaedic 
surgery. Our second experiment was thus designed to assess the ability of the proposed local phase-based technique to detect small gaps between bone fragments from 3D US data. First, the Sawbone was broken into two parts and each part was glued to the top surface of a metal block. For tracking purposes, infrared emitting diodes (IREDs) were glued onto the surface of one of the metal blocks. This part was tracked with an optical tracking system (OPTOTRAK 3020, Northern Digital Inc., Waterloo, ON, Canada with 3D localization accuracy of $0.1 \mathrm{~mm}$ in the directions parallel to the front of the camera) which was used to provide the gold standard displacement measurements. The other block with the second bone fragment was kept fixed. In total, 5 fixed displacements ranging from 0.8 to $2.2 \mathrm{~mm}$ in the vertical (Fig. $1 \mathrm{~d}$ ) and horizontal directions were then introduced. Tests were conducted with either a thick layer of ultrasound gel or a $3 \mathrm{~cm}$ thick slice of bovine tissue overlaid on the bone model. In both cases, the misalignment was tracked with the OPTOTRAK with a total of $103 \mathrm{D}$ US volumes obtained for each misalignment. The displacements along the top edges of the fracture boundaries were measured on each 2D slice of the $3 \mathrm{D}$ phase volume in which they appeared and then averaged. This result was then compared with the known applied displacement.

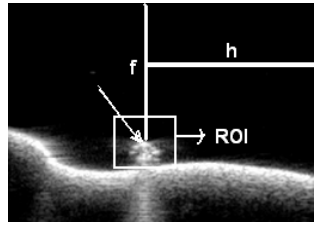

(a)

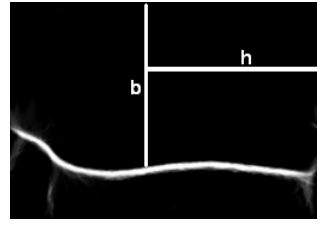

(b)

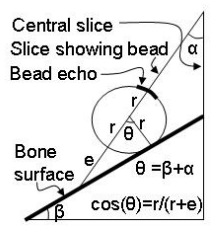

(c)

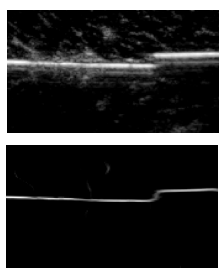

(d)

Fig. 1. Bone localization accuracy assessment (a-c: experiment 1, d: experiment 2). (a) Central slice of an US volume of a Sawbone. Arrow points to the fiducial (bead) attached to the bone. (b) Corresponding slice of 3D volume resulting from our proposed local phase processing. (c) For cases where the bead is not aligned with the central slice of the volume, the location of the bone surface can be calculated from the geometry of the angle of the plane showing the bead $(\alpha)$, the angle of the bone surface $(\beta)$ and the radius of the bead (r). (d) Central slice of an US volume(top) obtained by scanning phantom Sawbone bone fragments after introducing a vertical displacement and corresponding slice from our proposed method (bottom).

\section{Results}

\section{Experiment 1 Localization Accuracy:}

(A) Sawbone Specimen: The processing time was approximately 43s for each 3D volume. For both the horizontal and inclined specimens the mean error was calculated from the measurements taken at the 30 different bead locations for each depth setting. Among the three different depth settings the middle scanning depth resulted in a mean error of $0.62 \mathrm{~mm}(\mathrm{SD} 0.24 \mathrm{~mm})$ and $0.53 \mathrm{~mm}(\mathrm{SD} 0.28 \mathrm{~mm})$ inside the bone surface response on the 3DUS volume for the horizontal and inclined specimens, respectively. Compared to the other two depth settings these values were the highest error results obtained. 
(B) Bovine Specimen: For the cadaver experiment the mean localization error was $0.44 \mathrm{~mm}$ inside the bone surface response.

Experiment 2 Fracture Misalignment Detection: The mean errors in estimating fracture displacements were $-0.65 \mathrm{~mm}$ and $0.5 \mathrm{~mm}$ for horizontal and vertical misalignments, respectively, for the fractures imaged through bovine tissue. The error results obtained when the gel was used as an imaging medium were $-0.5 \mathrm{~mm}$ and $0.4 \mathrm{~mm}$ respectively.

Qualitative Results: Figure 2 shows a qualitative comparison of local-phaseprocessed images of a human distal radius with the original 3D US volume of the same bone. The local phase images are notably clearer than the 3DUS image, and the $3 \mathrm{D}$ version of the local phase images is markedly smoother than the $2 \mathrm{D}$ version, where each slice is treated independently in the latter.
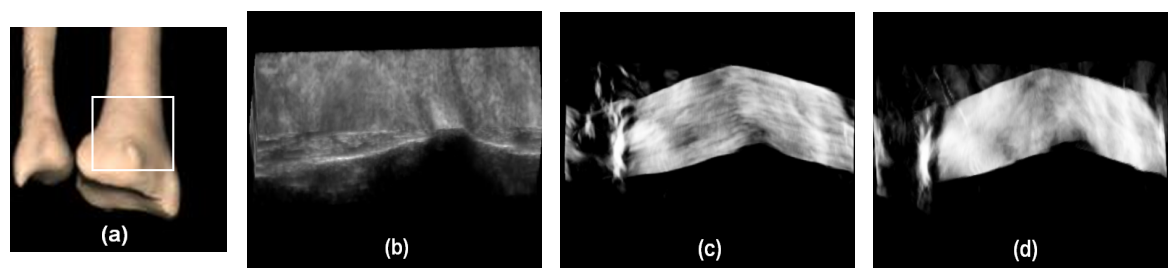

Fig. 2. Qualitative results on in-vivo human distal radius. (a) Area imaged. (b) Captured 3D US volume. (c) 2D phase feature image. (d) Proposed 3D phase symmetry image.

Figure 3 shows other qualitative examples where scans of an intact ex-vivo porcine tibia and fibula and fractured distal radius Sawbone were acquired. Note how the local phase processed images allow clear visualization of the entire bone surface and of the fracture line.

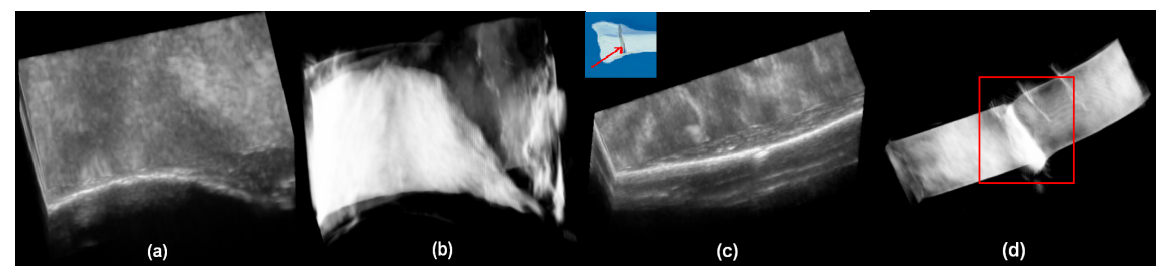

Fig. 3. Qualitative results obtained from ex vivo porcine tibia fibula specimen and Sawbone with soft tissue overlaid. (a) 3D US volume of intact bone. (b) Corresponding 3D phase symmetry image. (c) 3D US of a distal radius Sawbone fracture imaged with soft tissue overlaid on top. (d) 3D phase of (c) where the detected fracture is shown inside the red rectangle.

\section{Discussion and Conclusions}

In this paper we proposed a novel approach for accurate and fully automatic extraction of bone surfaces directly in 3D ultrasound volumes based on local phase symmetry 
image features that employ 3D Log-Gabor filters. Bone surface localization accuracy assessed using bone models and ex vivo porcine specimens showed a maximum mean error of $0.44 \mathrm{~mm}$ and a low standard deviation across the sampled points of only 0.16 $\mathrm{mm}$; these errors were relatively independent of the depth of the soft tissue/bone interface and of the inclination of the probe relative to the bone surface. Furthermore, the 3D phase method has high localization accuracy even when the US beam is not perfectly normal to the bone surface. Horizontal and vertical displacements between model bone fragments were also accurately measured with a maximum mean error under $0.65 \mathrm{~mm}$. The obtained results are encouraging for using local phase processed images in fracture assessment since the average accuracy required for such application is typically in the range of 2-4mm [13]. A comparison of in vivo scans of the human distal radius showed that a true $3 \mathrm{D}$ analysis produced a noticeably smoother image of the bone surface than previously reported $2 \mathrm{D}$ analysis. We expect that such 3D processing will be of special importance during the assessment of fractures where good accuracy is needed to avoid malunions. Furthermore, since there is no need to align the imaging plane with the anatomical area of interest, evaluation of the fractured area can likely be performed more rapidly.

We are currently investigating the performance of the method in volumes constructed using freehand 2D US and stitched 3D volumes where a larger region of interest can be obtained. Future work will also focus on establishing the feasibility of using the proposed technique for fracture assessment in the emergency department and for fracture reduction assessment in orthopaedic trauma applications, particularly of distal radius fractures.

\section{References}

1. Hanel, D.P., Jones, M.D., Trumble, T.E.: Wrist fractures. Orthop. Clin. North. Am. 33(1), 35-57 (2002)

2. Coppola, P.T., Coppola, M.: Emergency department evaluation and treatment of pelvic fractures. Emergency Medicine Clinics of North America 18(1), 1-27 (2000)

3. Jain, A.K., Taylor, R.H.: Understanding bone responses in B-mode ultrasound images and automatic bone surface extraction using a bayesian probabilistic framework. In: Proc. of SPIE Medical Imaging, pp. 131-142 (2004)

4. Barratt, D.C., Penney, P.G., Chan, S.K., Slomczykowski, M., Carter, T.J., Edwards, P.J., Hawkes, D.J.: Self calibrating 3D-ultrasound-based bone registration for minimally invasive orthopaedic surgery. IEEE Transactions on Medical Imaging 25, 312-323 (2006)

5. Kowal, J., Amstutz, C., Langlotz, F., Talib, H., Ballester, M.G.: Automated bone contour detection in ultrasound B-mode images for minimally invasive registration in computer assisted surgery an in vitro evaluation. The International Journal of Medical Robotics and Computer Assisted Surgery, 341-348 (2007)

6. Daanen, V., Tonetti, J., Troccaz, J.: A fully automated method for the delineation of osseous interface in ultrasound images. In: Barillot, C., Haynor, D.R., Hellier, P. (eds.) MICCAI 2004. LNCS, vol. 3216, pp. 549-557. Springer, Heidelberg (2004)

7. Amin, D.V., Kanade, T., Digioia, A.M., Jaramaz, B.: Ultrasound registration of the bone surface for surgical navigation. Journal of Computer Aided Surgery 8, 1-16 (2003)

8. Mulet-Parada, M., Noble, J.A.: 2D+T boundary detection in echocardiography. Medical Image Analysis 4(1), 21-30 (2000) 
9. Hacihaliloglu, I., Abugharbieh, R., Hodgson, A.J., Rohling, R.N.: Enhancement of bone surface visualization from 3D ultrasound based on local phase information. In: Proc. IEEE Ultrasonics Symposium, pp. 21-24 (2006)

10. Kovesi, P.: Symmetry and Asymmetry from Local Phase, AI 1997. In: Tenth Australian Joint Conference on Artificial Intellegence, pp. 185-190 (1997)

11. Dosil, R., Pardo, X.M., Fernandez-Vidal, X.R.: Data driven synthesis of composite feature detectors for 3D image analysis. Journal of Image and Vision Computing, 225-238 (2006)

12. Kovesi, P.: Image Features From Phase Congruency. Videre: A Journal of Computer Vision Research (1999)

13. Phillips, R.: The accuracy of surgical navigation for othopaedic surgery. Current Orthopaedics, 180-192 (2007) 